\title{
A Directional Antenna in a Matching Liquid for Microwave Radar Imaging
}

\author{
Saeed I. Latif, ${ }^{1}$ Daniel Flores Tapia, ${ }^{2,3}$ Diego Rodriguez Herrera, ${ }^{2}$ \\ Mario Solis Nepote, ${ }^{4}$ Stephen Pistorius, ${ }^{2,3,4}$ and Lotfollah Shafai ${ }^{5}$ \\ ${ }^{1}$ Department of Electrical and Computer Engineering, University of South Alabama, Mobile, AL 36688, USA \\ ${ }^{2}$ Department of Physics and Astronomy, University of Manitoba, 301 Allen Building, Winnipeg, MB, Canada R3T 2N2 \\ ${ }^{3}$ CancerCare Manitoba, 675 McDermot Avenue, Winnipeg, MB, Canada R3E 0V9 \\ ${ }^{4}$ Biomedical Engineering Program, University of Manitoba, E2-390 EITC, Winnipeg, MB, Canada R3T 5V6 \\ ${ }^{5}$ Department of Electrical and Computer Engineering, University of Manitoba, E2-390 EITC, Winnipeg, MB, Canada R3T 5V6
}

Correspondence should be addressed to Stephen Pistorius; stephen.pistorius@umanitoba.ca

Received 19 December 2014; Revised 15 October 2015; Accepted 19 October 2015

Academic Editor: Muhammad Ramlee Kamarudin

Copyright (C) 2015 Saeed I. Latif et al. This is an open access article distributed under the Creative Commons Attribution License, which permits unrestricted use, distribution, and reproduction in any medium, provided the original work is properly cited.

\begin{abstract}
The detailed design equations and antenna parameters for a directional antenna for breast imaging are presented in this paper. The antenna was designed so that it could be immersed in canola oil to achieve efficient coupling of the electromagnetic energy to the breast tissue. Ridges were used in the horn antenna to increase the operating bandwidth. The antenna has an exponentially tapered section for impedance matching. The double-ridged horn antenna has a wideband performance from $1.5 \mathrm{GHz}$ to $5 \mathrm{GHz}(3.75 \mathrm{GHz}$ or $110 \%$ of impedance bandwidth), which is suitable for breast microwave radar imaging. The fabricated antenna was tested and compared with simulated results, and similar bandwidths were obtained. Experiments were conducted on breast phantoms using these antennas, to detect a simulated breast lesion. The reconstructed image from the experiments shows distinguishable tumor responses indicating promising results for successful breast cancer detection.
\end{abstract}

\section{Introduction}

Breast cancer is the most common cancer worldwide in women. Approximately, 1.5 million women are diagnosed with breast cancer worldwide annually with North America and Europe having the highest incidence rate [1]. In 2014, an estimated 232,670 women will be diagnosed with breast cancer in the USA, with an estimated 40,000 deaths [2]. Developing countries have seen an increase in the number of cases and typically have even higher mortality rates. Early diagnosis and treatment are vital for the long-term survival of breast cancer patients. The current gold standard for breast cancer screening is X-ray mammography. Mammography is the current standard for the detection of breast cancer. Although this imaging modality has led to a decrease in mortality rates, the discomfort associated with breast compression, the use of ionizing radiation, and the 9- to 16-year cumulative false positive rates between 75 and $83 \%$ and a false negative rate between $7 \%$ and $9 \%$ allow room for improvement [3]. X-ray mammography also uses ionizing radiation, which limits the number of imaging procedures that can be performed on a subject due to the effects of cumulative exposure to radiation [4]. Magnetic Resonance Imaging (MRI) is also used for breast imaging, but it is expensive and not widely used for mass screening [4]. Another alternative is Ultrasound (US) imaging, but this is dependent on the availability of skilled operators.

Malignant breast tumors have dielectric constants and conductivities that are different to those of healthy breast tissues and Active Microwave Imaging (AMI) has the ability to detect these differences. It also has the potential to be a safe, quick, and less expensive tool for early detection of breast cancer $[5,6]$. The use of nonionizing radiation to illuminate the breast allows for more frequent investigations, and the responses collected from the breast structures enable breast lesions to be identified. Early studies show that microwave 
imaging has the potential to generate high contrast images, from which subcentimeter lesions can be detected [5, 7]. Two approaches to AMI are Microwave Tomography (MT) and breast microwave radar (BMR). The first approach requires the inversion of a nonlinear and ill-posed matrix and thus is computationally complex [7]. The second approach requires the use of ultra-wideband antennas to irradiate the target and receive the scattered signals to form a reflectivity map of the breast region [5]. BMR images are capable of identifying the location of strong scattering signatures that are likely to be a malignant lesion. BMR approaches are currently being tested in midscale clinical trials and the goal is to detect breast lesions of at least $4 \mathrm{~mm}$. Although progress in image resolution and $3 \mathrm{D}$ imaging and faster data acquisition have been made [8-10], hardware design and imaging algorithm still require improvements if this technology is to be used for routine clinical use. In particular, the antenna design is important, because the antenna directs the signal to the breast to be imaged, while antennas or other sensors receive the scattered signal. Low frequency signals have higher penetration depth and are more effective in interrogating breast tissue at large depths and hence improve the Signal-to-Noise Ratio (SNR). However, the antenna size increases at low frequencies, which is not desirable if a small and portable system is required. High frequency signals reduce the antenna size but have limited signal penetration in the skin and breast tissue. Therefore, a frequency range of $1.5 \mathrm{GHz}$ to $5 \mathrm{GHz}$ was chosen as the operating frequency for this application. Canola oil is an effective matching medium for coupling of electromagnetic signals into the breast [3]. Immersing the antennas in canola oil reduces the antenna size and this concept has been used to design several wideband antennas for breast microwave imaging $[5,6,11-18]$. High permittivity material, such as ceramic or distilled water, has been proposed for antenna miniaturization with distilled water as the matching liquid to obtain efficient coupling of the electromagnetic wave into the breast [17-19]. However, the high dissipation losses in distilled water reduce the coupling efficiency. Therefore, canola oil, a relatively low-loss and low permittivity material, was chosen as the matching medium for the system. In [20], a double-ridged horn antenna is proposed for operation in the $8-18 \mathrm{GHz}$ frequency band for wireless applications. In this paper, the design of a double-ridged horn antenna operating in the $1.5-5 \mathrm{GHz}$ band is presented.

The detailed antenna design and measured results are discussed in Section 2. Two antennas were used to conduct several experiments on synthetic phantoms that mimic the dielectric properties of breast tissues. Datasets were recorded for various situations and were used for image reconstruction and the results are presented in Section 3. Finally, in Section 4, the limitations and future research directions of the system are discussed.

\section{Antenna Design and Measurement Results}

Horn antennas provide directional radiation patterns that are optimised for the proposed breast microwave radar system. The directionality is an important feature that can increase the energy scattered by the tumor and SNR. To maximize

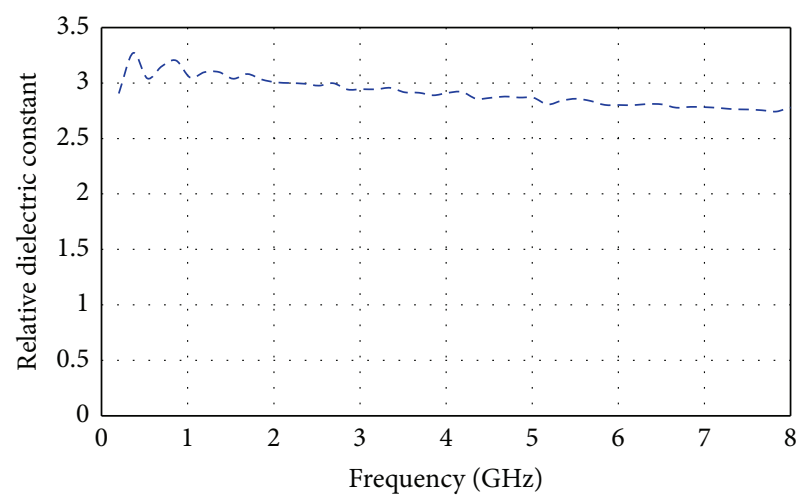

FIGURE 1: The variation of the dielectric constant with frequency for the canola oil.

the coupling efficiency, the antenna must be matched to the breast and should have minimal dissipation and spillover; that is, it must not radiate away from the breast to be imaged. The latter requirement maximizes the power coupling into the tissue and minimizes the parasitic coupling between the transmitting and receiving antennas. Parasitic coupling can increase the noise floor of the measurement system and creates clutter and degrades the image [4]. Horn antennas have directional radiation patterns and wide impedance bandwidth. The impedance bandwidth can be further increased using ridges in the waveguide and flared sections [17-20]. For the chosen frequency of $1.5-5 \mathrm{GHz}$, the double-ridged horn antenna is designed using the methodology presented in [20]. This horn antenna consists of several waveguide sections, where ridges are introduced into the $E$-plane to lower the cutoff frequency of the waveguide. As canola oil is the matching liquid for the project, this is incorporated into the antenna design. An antenna in free space experiences the free-space wave impedance of

$$
Z=\sqrt{\frac{\mu_{o}}{\varepsilon_{o}}},
$$

where $\varepsilon_{o}=8.854 \times 10^{-12}$ farads per meter $\left(\mathrm{F} \cdot \mathrm{m}^{-1}\right)$ and $\mu_{o}=$ $4 \pi \times 10^{-7} \mathrm{~V} \cdot \mathrm{s} /(\mathrm{A} \cdot \mathrm{m}) . Z$ is $377 \Omega$. If the antenna is immersed in canola oil, the impedance seen by the antenna is different and can be calculated using the following equation:

$$
Z_{\text {oil }}=\sqrt{\frac{\mu_{o}}{\varepsilon_{o} \varepsilon_{r}}} .
$$

Canola oil's material properties were measured in the Antenna Lab at the University of Manitoba. The dielectric properties of canola oil used in the system were measured using an Agilent 85070d dielectric probe measuring kit. The measured relative dielectric permittivity of the canola oil is shown as a function of frequency in Figure 1. It is evident that although it varies with frequency, the relative dielectric permittivity of canola oil is approximately 3 at $2 \mathrm{GHz}$. Other properties of the canola oil are conductivity, $\sigma=0.03 \mathrm{~S} / \mathrm{m}$, and loss tangent, $\tan \delta=0.08$. Substitution of canola oil's 


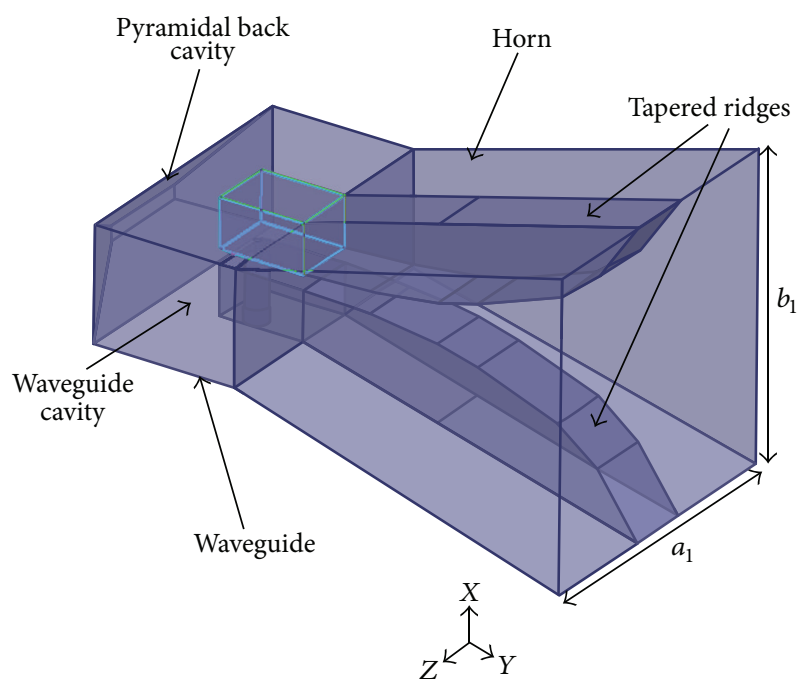

(a)

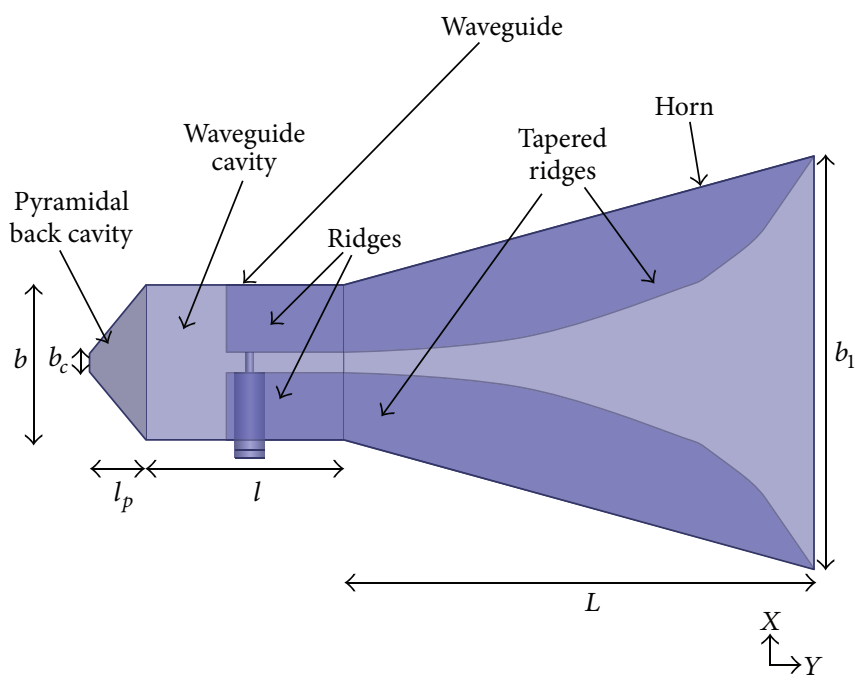

(b)

FIgURE 2: Geometry of the double-ridged horn antenna designed for breast microwave imaging system; (a) 3D view and (b) side view.

dielectric properties into (2) gives $Z_{\text {oil }}=217 \Omega$. The doubleridged horn antenna geometry, shown in Figure 2, is designed to match this impedance. The antenna has two main parts: the waveguide section and the horn section. The flare of the horn section, with ridges, matches the $50 \Omega$ input impedance to the impedance at the opening of the horn, which in canola oil is $217 \Omega$. The flare section contains 7 ridged waveguide sections, whose dimensions are governed by the following equation to obtain the appropriate impedance matching:

$$
Z(y)=Z_{o} e^{k y}, \quad(0 \leq y \leq L) .
$$

Here, $Z(y)$ is the impedance at a distance $y$ from the waveguide aperture and $L$ is the total axial length, of $70 \mathrm{~mm}$, with the flared section having an exponential taper. $k$ is calculated using the following:

$$
k=\frac{1}{L} \ln \frac{Z_{\text {oil }}}{Z_{o}},
$$

where $Z_{o}$ and $Z_{\text {oil }}$ are the characteristic impedances of the double-ridged rectangular waveguide and the canola oil, respectively. The first waveguide, which is connected to the $50 \Omega \mathrm{SMA}$, is $29.4 \mathrm{~mm}$ long and has a characteristic impedance of $50 \Omega$. The dimensions of the subsequent waveguides and corresponding ridges are obtained using (3)-(4) and tabulated in Table 1 . The antenna has a pyramidal back cavity for further impedance matching, as shown in Figure 2. Other antenna parameters are given in Table 2 (the parameters are labeled in Figure 2). The aperture size of the horn antenna is $61.6 \mathrm{~mm} \times 61.6 \mathrm{~mm}$ and overall length is $108 \mathrm{~mm}$.

Two antenna prototypes were fabricated and tested in the Antenna Lab and the Physics Lab at the University of Manitoba. A photograph of the fabricated antenna is shown in Figure 3. The simulated and measured reflection coefficient plots are compared in Figure 4(a). The simulation results were obtained using Ansys HFSS, version 14.0 [21]. Both measured
TABLE 1: Details of the waveguide section: waveguide (WG) size and ridge height.

\begin{tabular}{lccc}
\hline $\begin{array}{l}\text { Waveguide } \\
\text { section }\end{array}$ & $\begin{array}{c}\text { Waveguide } \\
\text { length }(\mathrm{mm})\end{array}$ & $\begin{array}{c}\text { Waveguide } \\
\text { height }(\mathrm{mm})\end{array}$ & $\begin{array}{c}\text { Ridge height } \\
(\mathrm{mm})\end{array}$ \\
\hline 1 & 29.4 & 23.3 & 10.0 \\
2 & 14 & 31 & 13.3 \\
3 & 14 & 38.6 & 15.4 \\
4 & 8.4 & 43.2 & 15.4 \\
5 & 8.4 & 47.8 & 14.7 \\
6 & 8.4 & 52.4 & 14 \\
7 & 8.4 & 57 & 9.8 \\
8 & 8.4 & 61.6 & 0 \\
\hline
\end{tabular}

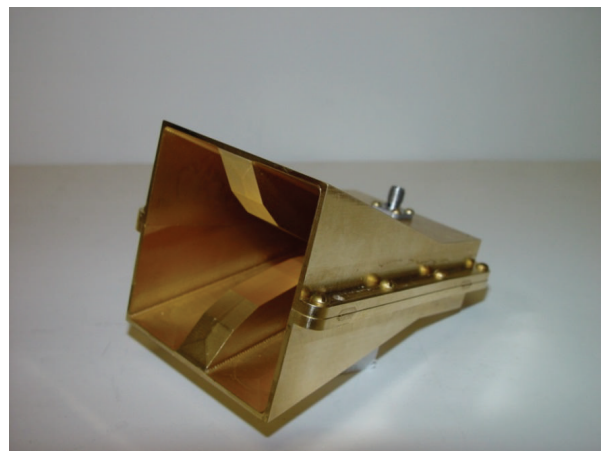

FIGURE 3: Fabricated double-ridged horn antenna prototype.

and simulated results of the antenna, immersed in canola oil, exhibit a $-10 \mathrm{~dB} S_{11}$ bandwidth of $100 \%$. If $S_{11}=-7 \mathrm{~dB}$ is considered as the reference, the measured bandwidth of this antenna is $110 \%(1.54 \mathrm{GHz}$ to $5.29 \mathrm{GHz})$. In the BMR approach, the transmission response is of great importance, as it indicates the signal propagation and scattering from 


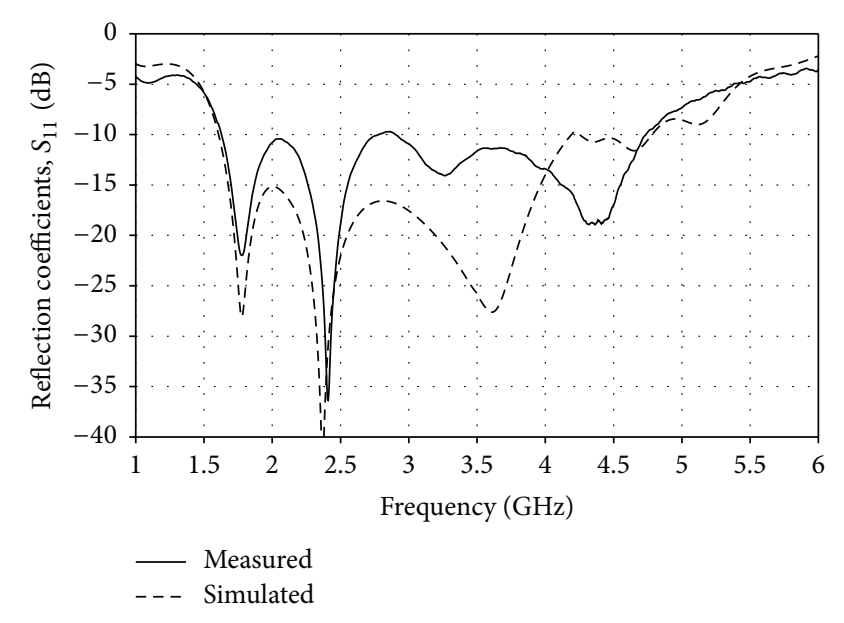

(a)

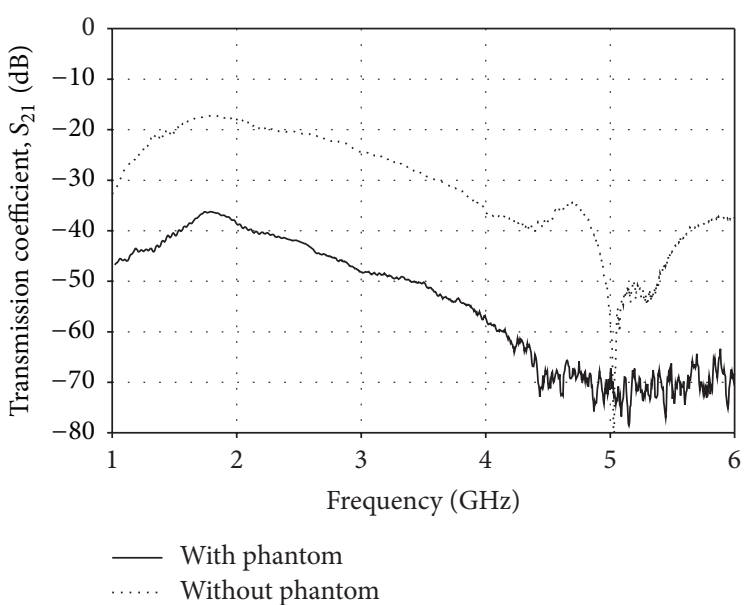

(b)

Figure 4: (a) Simulated and measured reflection coefficient of the double-ridged horn antenna designed for medical imaging. (b) Transmission coefficients of the antenna with and without breast phantom. Glycerin was used as the breast phantom material.

TABLE 2: The double-ridged horn antenna parameters.

\begin{tabular}{llc}
\hline Section & Parameters & Dimension \\
\hline & Waveguide width, $a^{*}$ & $56 \mathrm{~mm}$ \\
& Waveguide height, $b$ & $23.2 \mathrm{~mm}$ \\
Waveguide & Waveguide length, $l$ & $29.4 \mathrm{~mm}$ \\
& Ridge width & $13 \mathrm{~mm}$ \\
& Ridge height & $10 \mathrm{~mm}$ \\
& Ridge length & $17.5 \mathrm{~mm}$ \\
\hline \multirow{4}{*}{$\begin{array}{l}\text { Pyramidal back } \\
\text { cavity }\end{array}$} & Cavity length, $l_{p}$ & $8.4 \mathrm{~mm}$ \\
& In the waveguide side & \\
& Cavity width, $a^{*}$ & $56 \mathrm{~mm}$ \\
& Cavity height, $b$ & $23.3 \mathrm{~mm}$ \\
& Cavity width & \\
Cavity height, $b_{c}$ & $19.6 \mathrm{~mm}$ \\
& Horn length, $L$ & $2.8 \mathrm{~mm}$ \\
\hline In the waveguide side \\
Width, $a^{*}$
\end{tabular}

${ }^{*} a$ is not shown in Figure 2(b).

the breast tissue. The transmission response of the designed double-ridged horn antenna was evaluated by immersing two antennas in canola oil, facing each other and separated by a distance of $190 \mathrm{~mm}$. As the antennas are designed for breast imaging, a breast phantom was introduced between the antennas to predict their performance in the presence of a breast. Low-density breast tissue was simulated using glycerin since its dielectric permittivity mimics the average values found in low-density breast regions. The breast phantom was a styrene-acrylonitrile cylinder with a diameter of $130 \mathrm{~mm}$

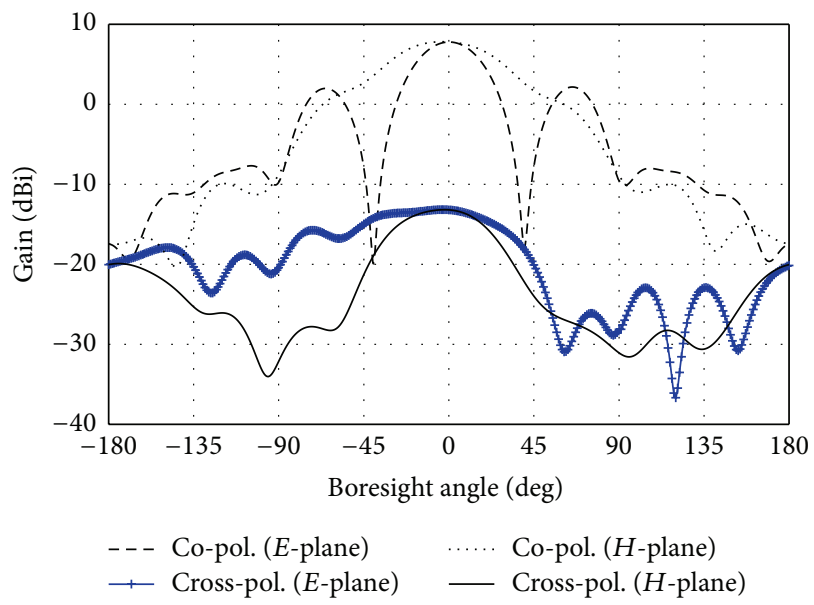

Figure 5: Radiation patterns of the designed double-ridged horn antenna in two principal planes at $2.4 \mathrm{GHz}$.

and wall thickness of $2 \mathrm{~mm}\left(0.01 \lambda_{o}\right.$, where $\lambda_{o}$ is the free-space wavelength at $1.5 \mathrm{GHz}$ ), filled with glycerin. The cylinder wall was $30 \mathrm{~mm}$ from the antenna apertures. In canola oil, the reactive zone of the antenna is $28 \mathrm{~mm}$ long. The measured results are shown in Figure 4(b). In canola oil, the antenna showed a peak transmission response of $-18 \mathrm{~dB}$ at $1.75 \mathrm{GHz}$, with $S_{21}$ better than $-40 \mathrm{~dB}$ being observed up to $4.87 \mathrm{GHz}$. In the presence of the phantom, a peak $S_{21}$ value of only $-30 \mathrm{~dB}$ was recorded, due to the losses in the breast phantom material. An $S_{21}$ of better than $-60 \mathrm{~dB}$ up to $4 \mathrm{GHz}$ was measured. The variation in $S_{21}$ due to the presence of the phantom was recorded for image processing.

The radiation patterns of the antenna in two principal planes at $2.4 \mathrm{GHz}$ are shown in Figure 5. The antenna has a narrow beamwidth in the $H$-plane ( $y z$ plane) with some sidelobes, compared to that in the $E$-plane ( $x y$ plane). Due to the loss in the coupling medium, the antenna has a moderate peak gain of $7.8 \mathrm{dBi}$ at $2.4 \mathrm{GHz}$ in the boresight direction. 


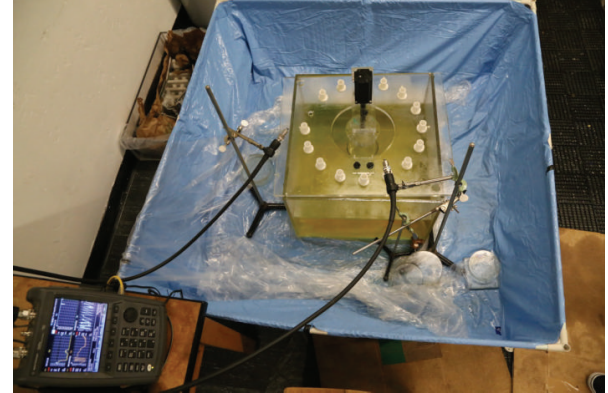

Figure 6: Photograph of the measurement setup showing the stepper motor to rotate the phantom and holders to position the transmitting and receiving antennas. [The antennas are not attached to holders in this photograph.]

\section{Experiments for Breast Tumor Detection}

The horn antennas were attached to the posterior wall of a $56 \times 56 \times 40 \mathrm{~cm}$ Plexiglas tank, and the BMR datasets were recorded using a data acquisition system. The tank with the rotational mechanism is shown in Figure 6. The tank was filled with canola oil to provide impedance matching between the antenna and the breast phantom. In the experiment, bistatic $\left(S_{21}\right)$ radar responses were recorded using an Agilent N9923A vector network analyzer, which functioned as both the microwave waveform generator and the recording device. When measuring the bistatic responses, the angular separation between the transmitter and the receiver antennae was $135^{\circ}$. This value was obtained experimentally by searching for the separation that minimized the magnitude of the cross talk between the transmitter and receiver antennae. A 130-mm diameter cylinder with a height of $35 \mathrm{~cm}$, filled with glycerin, was used as the phantom, which was located outside the reactive zone of the antenna. The phantom was positioned in the center of the tank and rotated to perform the data acquisition process. Tumors were simulated using spherical capsules of $8 \mathrm{~mm}$ diameter filled with an $85 \%$ saline and $15 \%$ fructose solution. This mixture was chosen based on the dielectric characteristics of the components as studied by Shinyashiki et al. [22]. Synthetic fibroglandular structures were introduced using a mixture of $25 \%$ synthetic polymer compound and $75 \%$ distilled water. The inclusion of fibroglandular regions is essential, because, in $80 \%$ of cases, breast tumors occur next to dense tissue structures. The dielectric properties of the breast tissues and materials used in the experimental setup are given in Table 3. The datasets were collected along a circular scan geometry with a radius of $16 \mathrm{~cm}$. The phantom was positioned in the center of the system. The synthetic fibroglandular structures were attached to acrylic rods that were connected to a support base. This support base was attached via a nylon screw to the shaft of a stepper motor. This motor was used to rotate the antenna to a discrete set of positions to emulate a circular scan geometry. In this study, all datasets were collected by positioning the phantom at 144 different angles, which emulates 144 different scan locations. After the phantom contents had been rotated into a particular angular position, a stepped frequency continuous wave with
TABLE 3: Dielectric properties of breast tissues and the materials used in the experimental setup.

\begin{tabular}{lcc}
\hline Material & $\varepsilon_{r}(3 \mathrm{GHz})$ & $\sigma(3 \mathrm{GHz})$ \\
\hline Skin & 35 & $1.2 \mathrm{~S} / \mathrm{m}$ \\
Low-density breast tissue & 9 & $0.4 \mathrm{~S} / \mathrm{m}$ \\
Glycerin & 8.9 & $0.72 \mathrm{~S} / \mathrm{m}$ \\
Fibroglandular tissue & $25-32$ & $1.5 \mathrm{~S} / \mathrm{m}$ \\
Polymer-distilled water mixture & $30-36$ & $1.6 \mathrm{~S} / \mathrm{m}$ \\
Tumor & 55 & $2.1 \mathrm{~S} / \mathrm{m}$ \\
Fructose-saline mixture & 53 & $1.9 \mathrm{~S} / \mathrm{m}$ \\
Canola oil & 2.5 & $0.035 \mathrm{~S} / \mathrm{m}$ \\
Styrene-acrylonitrile & 2.6 & $0.001 \mathrm{~S} / \mathrm{m}$ \\
\hline
\end{tabular}

a bandwidth of $5.98 \mathrm{GHz}$ and a center frequency of $3.01 \mathrm{GHz}$ was applied. Each waveform had 601 frequency points. The energy of each frequency component in the irradiated wave was $1 \mathrm{~mW}$. The datasets were used for image reconstruction using the multistatic approach described in [23].

The quality of the reconstructed datasets was quantitatively assessed by calculating the spatial accuracy, Signalto-Clutter Ratio (SCR), Contrast-to-Clutter Ratio (CCR), and Tumor-to-Fibroglandular Response Ratio (TFRR) of the reconstructed images. The SCR is defined as

$$
\mathrm{SCR}=20 \cdot \log _{10}\left(\frac{\Gamma}{\sigma_{b}}\right) .
$$

In (5), $\Gamma$ is the maximum magnitude of the tumor signature and $\sigma_{b}$ is the standard deviation of the background. It was calculated as follows:

$$
\text { TFER }=20 \cdot \log _{10}\left(\frac{\Gamma}{\Theta}\right),
$$

where $\Theta$ is the maximum magnitude of the fibroglandular structures signatures. The third metric, CCR, is defined as follows:

$$
\mathrm{CCR}=10 \cdot \log _{10}\left(\frac{\left(\Gamma^{2}-\Theta^{2}\right)}{\sigma_{b}}\right)
$$

This definition is used in the case of CCR since the energy of the reconstructed images is commonly used to assess the presence of any lesions [24-26].

The first experimental setup with fibroglandular tissues is shown in Figure 7. This setup does not include any tumors. The reconstructed image is shown in Figure 8. In all the reconstructed images, the center of the phantom was denoted as the origin. Note that the location of the dense tissue structure signatures is consistent with their physical location. A second experimental setup is shown in Figure 9. This setup includes a tumor on the dextral side of the dense tissue structure. The targets were located at the same height as the antenna center. The reconstructed image from the datasets is shown in Figure 10. Regions of fibroglandular tissue are typically larger than tumors and have a contrast of 2-1.1 with respect to the fatty tissues, which is less than that of malignant lesions, whose contrast to fatty tissues ranges from 10 to $5: 1$. 


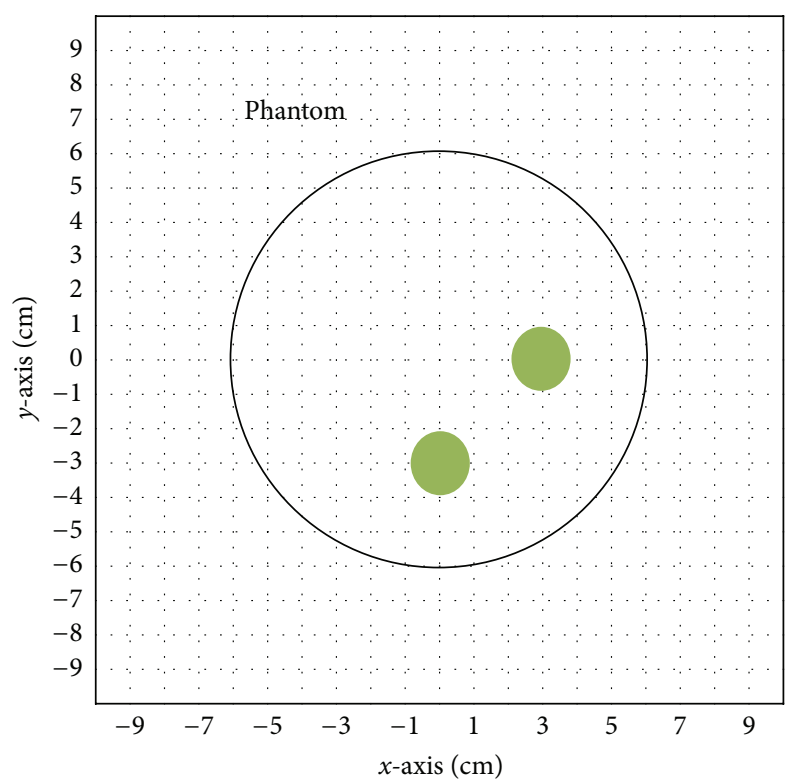

Figure 7: Experiment 1 setup. The fibroglandular structures are denoted in green.

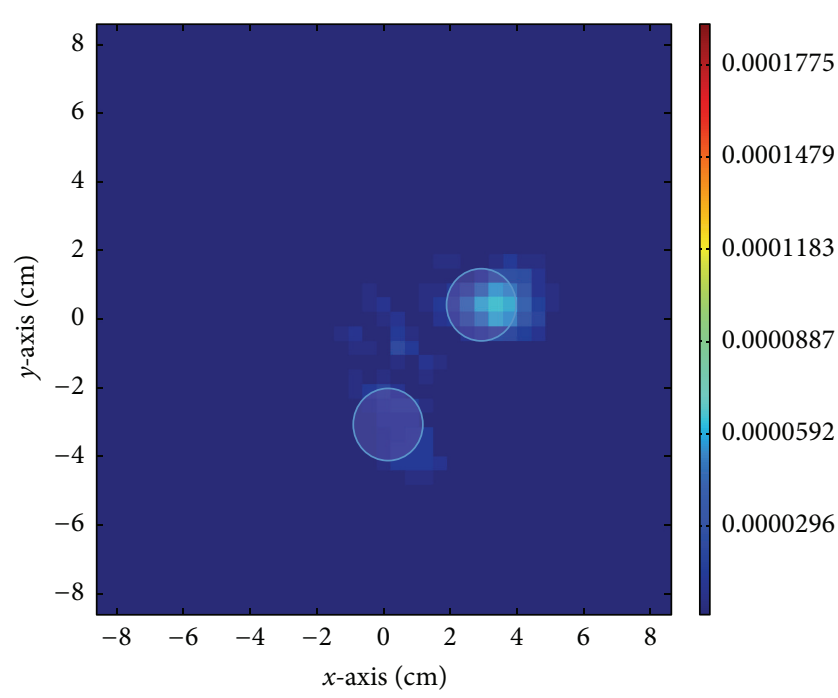

FIGURE 8: Reconstructed image from the multistatic dataset recorded from the experimental setup shown in Figure 7. The green circles denote the location of the dense tissue structures.

However, the presence of dense fibroglandular regions near the tumor can make it difficult to visualize the tumor signatures in the reconstructed images. Figure 10 illustrates that, with this combination of antennas, phantom geometry, and reconstruction algorithm, the tumor responses have a magnitude that is three times larger than the adjacent dense tissue structures. A compendium of the performance metrics for the reconstructed images can be found in Tables 4 and 5. For both datasets, the spatial accuracy of the reconstructed dense tissue and tumor signatures was within the spatial resolution of the reconstructed images $(8 \mathrm{~mm})$. Compared to published data from several experimental setups in [24-26], the results

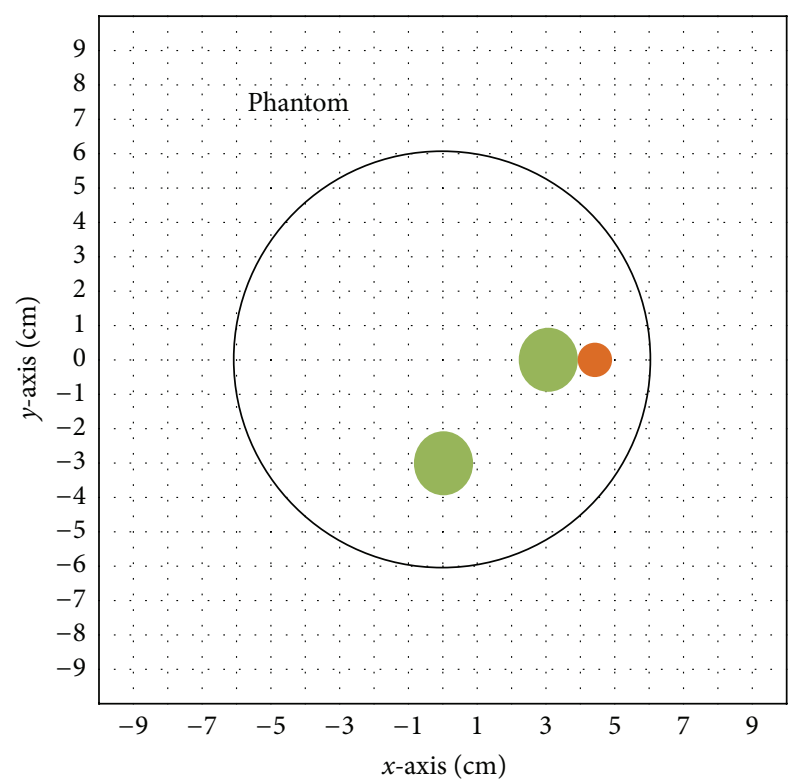

FIgURE 9: Experiment 2 setup. The fibroglandular structures are denoted in green and tumor is denoted in red.

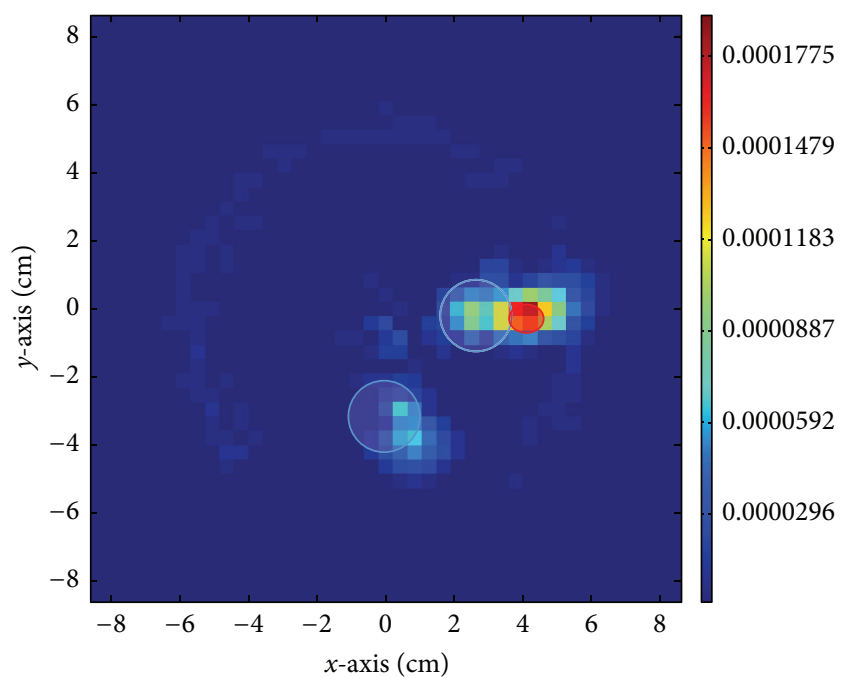

FIGURE 10: Reconstructed image from the multistatic dataset recorded from the experimental setup shown in Figure 9. The green circles denote the location of the dense tissue structures and red circle denotes the location of the synthetic tumor.

obtained using the antenna proposed in this paper exhibit higher SCR values and similar spatial accuracy.

\section{Conclusions}

The design and testing of a double-ridged horn antenna are presented in this paper. The antenna was designed to be immersed in canola oil to provide optimal antenna-breast coupling for breast microwave radar-based imaging. The simulated measured reflection and transmission responses are discussed. The antenna shows $S_{11}=-7 \mathrm{~dB}$ bandwidth of $110 \%$ with a favorable transmission response. Experiments were 
TABLE 4: Imaging performance metrics for each reconstructed image.

\begin{tabular}{lccc}
\hline Dataset/metric & TFR $(\mathrm{dB})$ & SCR $(\mathrm{dB})$ & $\mathrm{CNR}(\mathrm{dB})$ \\
\hline No tumor & N/A & 7.7 & N/A \\
Tumor & 4.1 & 12.2 & 3.0 \\
\hline
\end{tabular}

TABLE 5: Spatial accuracy of the reconstructed images.

\begin{tabular}{lcc}
\hline Dataset/tissue type & Fibroglandular & Tumor \\
\hline No tumor & $(4,1) \mathrm{mm}$ & N/A \\
Tumor & $(4,0) \mathrm{mm}$ & $(3,0) \mathrm{mm}$ \\
\hline
\end{tabular}

conducted to evaluate the performance of the antenna, by using various mixtures to mimic an inhomogeneous breast with fibroglandular tissue and tumor. The image reconstructed using the datasets recorded from the experimental setup with the double-ridged horn antennas successfully recovers the differentiable response of a simulated $8 \mathrm{~mm}$ tumor at a location consistent with its position in the experimental setup, even in the presence of dense fibroglandular tissues. The wideband property and the effective transmission response of the horn antenna will be useful for the development of a complete microwave imaging system.

\section{Conflict of Interests}

The authors declare that there is no conflict of interests regarding the publication of this paper.

\section{Acknowledgments}

The authors would like to thank Cory Smith and Brad Tabachnick for their help in the antenna fabrication and testing. This work was done at the University of Manitoba and CancerCare Manitoba. Financial support by the Canadian Breast Cancer Foundation, the National Science and Engineering Research Council of Canada, and CancerCare Manitoba is gratefully acknowledged.

\section{References}

[1] K. Servick, "Breast cancer: a world of differences," Science, vol. 343, no. 6178, pp. 1452-1453, 2014.

[2] R. Siegel, J. Ma, Z. Zou, and A. Jemal, "Cancer statistics, 2014," CA: A Cancer Journal for Clinicians, vol. 64, no. 1, pp. 9-29, 2014.

[3] K. K. Jacobsen, E. S. O’Meara, D. Key et al., “Comparing sensitivity and specificity of screening mammography in the United States and Denmark," International Journal of Cancer, vol. 137, no. 9, pp. 2198-2207, 2015.

[4] N. K. Nikolova, "Microwave imaging for breast cancer," IEEE Microwave Magazine, vol. 12, no. 7, pp. 78-94, 2011.

[5] M. Klemm, I. J. Craddock, J. A. Leendertz, A. Preece, and R. Benjamin, "Radar-based breast cancer detection using a hemispherical antenna array-experimental results," IEEE Transactions on Antennas and Propagation, vol. 57, no. 6, pp. 1692-1704, 2009.
[6] R. J. Halter, T. Zhou, P. M. Meaney et al., “The correlation of in vivo and ex vivo tissue dielectric properties to validate electromagnetic breast imaging: initial clinical experience," Physiological Measurement, vol. 30, no. 6, pp. S121-S136, 2009.

[7] T. M. Grzegorczyk, P. M. Meaney, P. A. Kaufman, R. M. Diflorio-Alexander, and K. D. Paulsen, "Fast 3-D tomographic microwave imaging for breast cancer detection," IEEE Transactions on Medical Imaging, vol. 31, no. 8, pp. 1584-1592, 2012.

[8] N. R. Epstein, P. M. Meaney, and K. D. Paulsen, "3D paralleldetection microwave tomography for clinical breast imaging," Review of Scientific Instruments, vol. 85, no. 12, Article ID 124704, 2014.

[9] M. Asefi, M. OstadRahimi, A. Zakaria, and J. LoVetri, "A 3$\mathrm{D}$ dual-polarized near-field microwave imaging system," IEEE Transactions on Microwave Theory and Techniques, vol. 62, no. 8, pp. 1790-1797, 2014.

[10] D. Byrne and I. J. Craddock, "Time-domain wideband adaptive beamforming for radar breast imaging," IEEE Transactions on Antennas and Propagation, vol. 63, no. 4, pp. 1725-1735, 2015.

[11] D. Gibbins, M. Klemm, I. J. Craddock, J. A. Leendertz, A. Preece, and R. Benjamin, "A comparison of a wide-slot and a stacked patch antenna for the purpose of breast cancer detection," IEEE Transactions on Antennas and Propagation, vol. 58, no. 3, pp. 665-674, 2010.

[12] J. Bourqui, M. Okoniewski, and E. C. Fear, "Balanced antipodal vivaldi antenna with dielectric director for near-field microwave imaging," IEEE Transactions on Antennas and Propagation, vol. 58, no. 7, pp. 2318-2326, 2010.

[13] M. A. Campbell, M. Okoniewski, and E. C. Fea, "TEM horn antenna for near-field microwave imaging," Microwave and Optical Technology Letters, vol. 52, no. 5, pp. 1164-1170, 2010.

[14] S. I. Latif, S. Pistorius, L. Shafai, and D. Flores-Tapia, "A planar ultrawideband elliptical monopole antenna with reflector for breast microwave imaging," Microwave and Optical Technology Letters, vol. 56, no. 4, pp. 808-813, 2014.

[15] X. Li, S. C. Hagness, M. K. Choi, and D. W. Van Der Weide, "Numerical and experimental investigation of an ultrawideband ridged pyramidal horn antenna with curved launching plane for pulse radiation," IEEE Antennas and Wireless Propagation Letters, vol. 2, pp. 259-262, 2003.

[16] R. K. Amineh, A. Trehan, and N. K. Nikolova, “TEM horn antenna for ultra-wide band microwave breast imaging," Progress In Electromagnetics Research B, no. 13, pp. 59-74, 2009.

[17] U. Schwarz, R. Stephan, and M. A. Hein, "Experimental validation of high-permittivity ceramic double-ridged horn antennas for biomedical ultra-wideband diagnostics," in Proceedings of the IEEE International Conference on Wireless Information Technology and Systems (ICWITS '10), pp. 1-4, IEEE, Honolulu, Hawaii, USA, September 2010.

[18] S. I. Latif, D. Flores-Tapia, S. Pistorius, and L. Shafai, "Design and performance analysis of the miniaturised water-filled double-ridged horn antenna for active microwave imaging applications," IET Microwaves, Antennas \& Propagation, vol. 9, no. 11, pp. 1173-1178, 2015.

[19] F. Scotto di Clemente, R. Stephan, U. Schwarz, and M. A. Hein, "Miniature body-matched double-ridged horn antennas for biomedical UWB imaging," in Proceedings of the IEEE-APS Topical Conference on Antennas and Propagation in Wireless Communications (APWC '12), pp. 574-577, IEEE, Cape Town, South Africa, September 2012. 
[20] A. R. Mallahzadeh and A. Imani, "Double-ridged antenna for wideband applications," Progress in Electromagnetics Research, vol. 91, pp. 273-285, 2009.

[21] Ansoft HFSS, version 10, USA.

[22] N. Shinyashiki, M. Shinohara, Y. Iwata et al., "The glass transition and dielectric secondary relaxation of fructose-water mixtures," The Journal of Physical Chemistry B, vol. 112, no. 48, pp. 15470-15477, 2008.

[23] D. F. Tapia, D. R. Herrera, M. S. Nepote, A. Maizlish, C. M. Alabaster, and S. Pistorius, "Holographic reconstruction of multistatic breast microwave radar images: initial results on synthetic phantoms," in Proceedings of the 15th International Conference on Electromagnetics in Advanced Applications (ICEAA '13), pp. 1103-1106, IEEE, Torino, Italy, September 2013.

[24] M. Klemm, J. A. Leendertz, D. Gibbins, I. J. Craddock, A. Preece, and R. Benjamin, "Microwave radar-based breast cancer detection: imaging in inhomogeneous breast phantoms," IEEE Antennas and Wireless Propagation Letters, vol. 8, pp. 1349-1352, 2009.

[25] J. M. Sill and E. C. Fear, "Tissue sensing adaptive radar for breast cancer detection-experimental investigation of simple tumor models," IEEE Transactions on Microwave Theory and Techniques, vol. 53, no. 11, pp. 3312-3319, 2005.

[26] S. M. Salvador and G. Vecchi, "Experimental tests of microwave breast cancer detection on phantoms," IEEE Transactions on Antennas and Propagation, vol. 57, no. 6, pp. 1705-1712, 2009. 

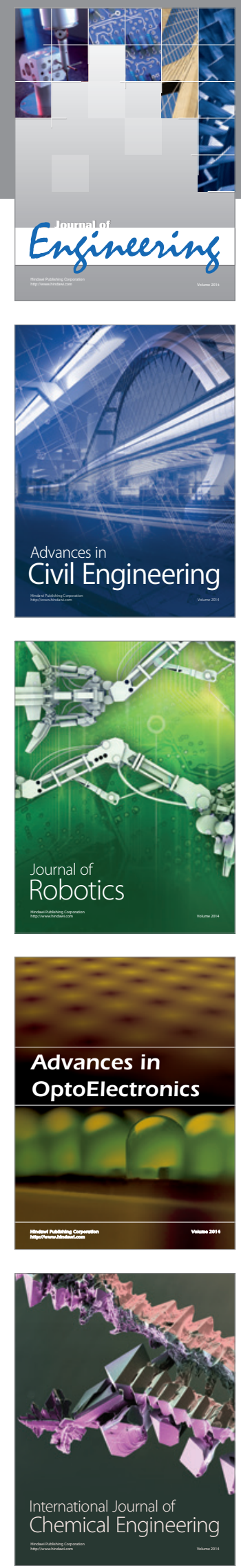

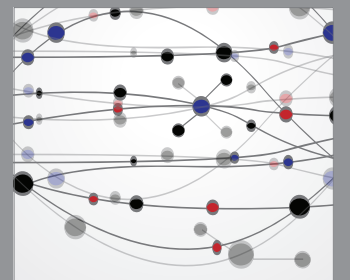

The Scientific World Journal
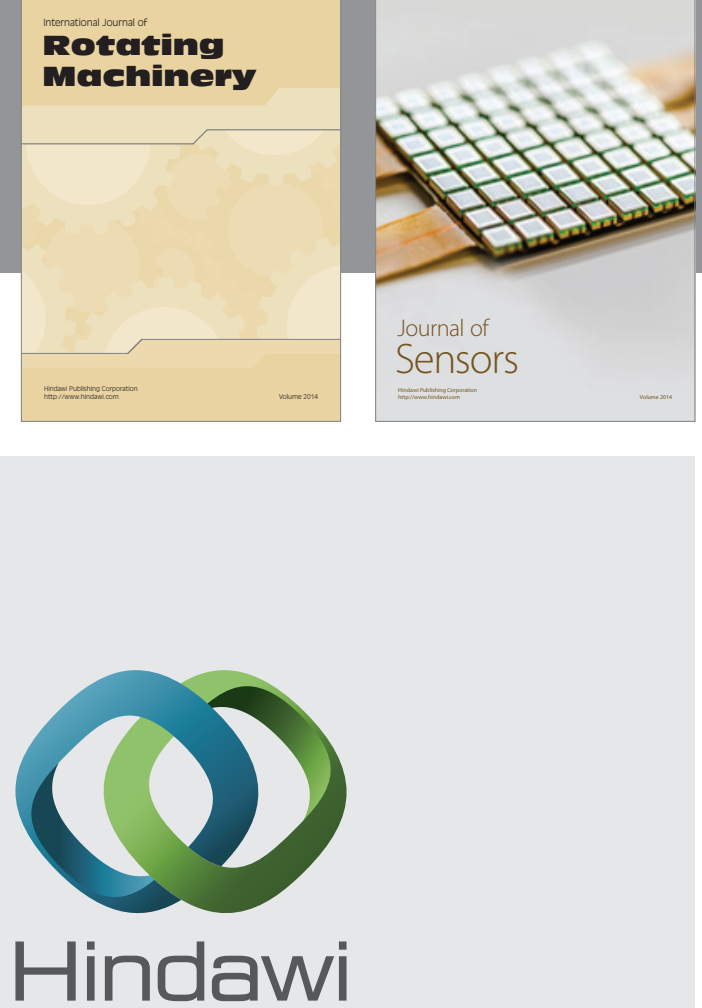

Submit your manuscripts at http://www.hindawi.com
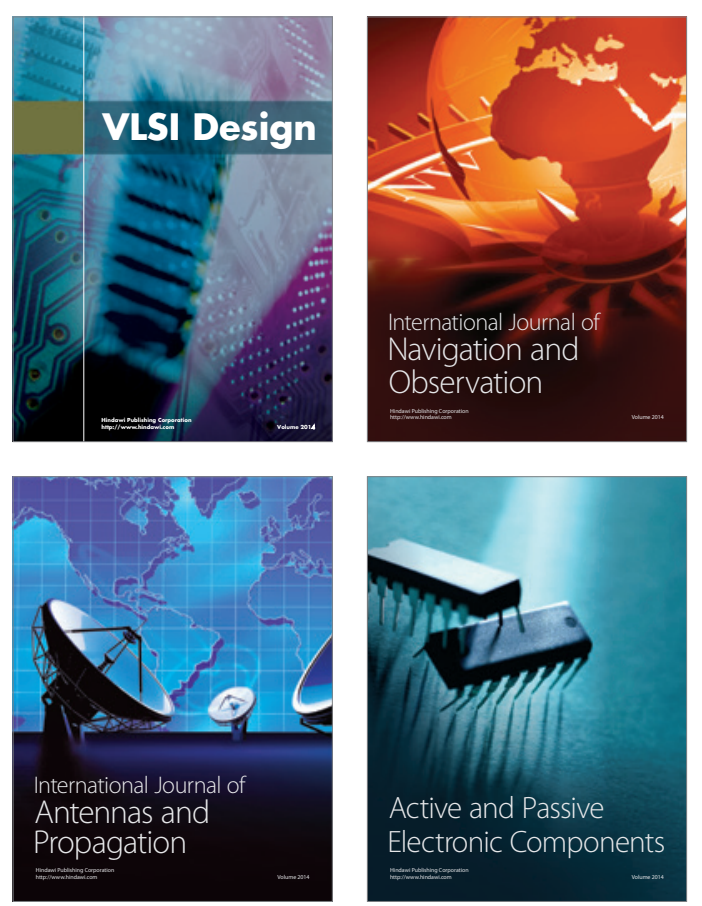
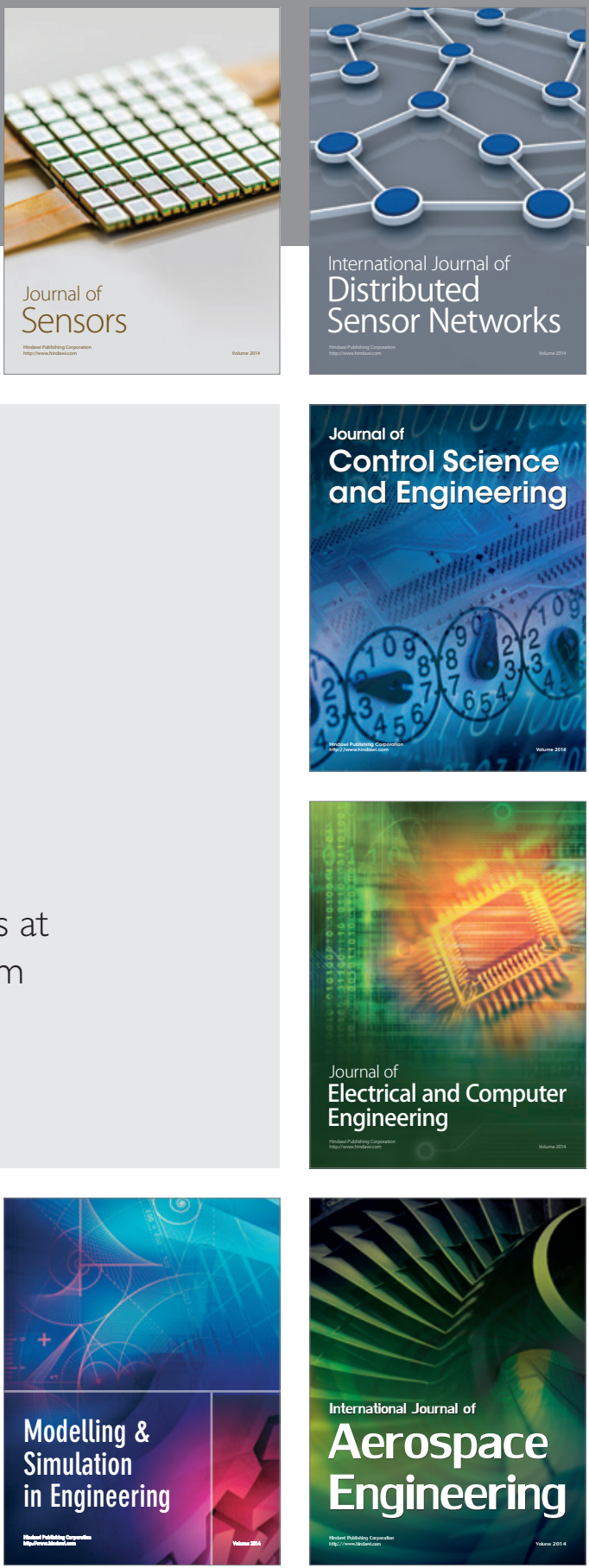

Journal of

Control Science

and Engineering
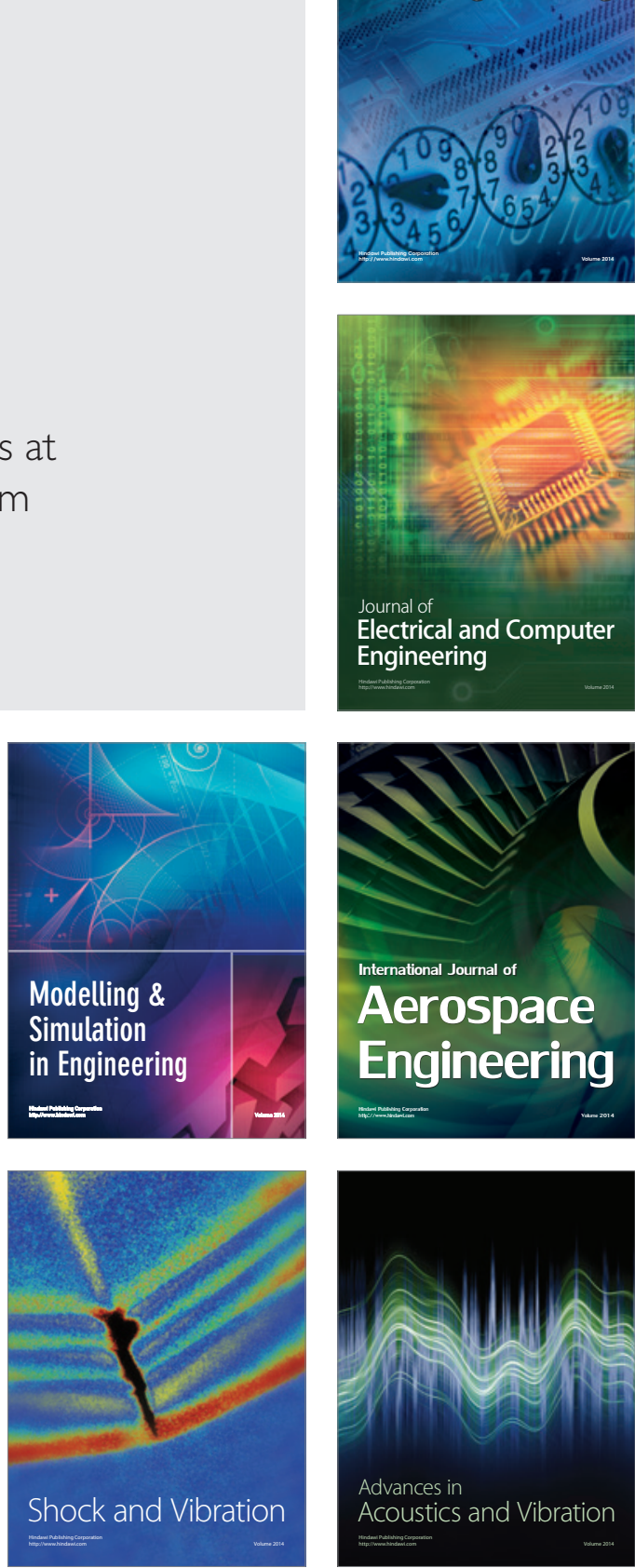